\title{
Safety and Efficacy of Chloral Hydrate in Outpatient Pediatrics for Sedation
}

\author{
Ruijuan Qiu, Guohua Huang* \\ Central Injection Room, The First Affiliated Hospital of Jinan University, Guangzhou, China \\ Email address: \\ hqyyq60304@163.com (Ruijuan Qiu),jndxhgh@163.com (Guohua Huang) \\ ${ }^{*}$ Corresponding author
}

\section{To cite this article:}

Ruijuan Qiu, Guohua Huang. Safety and Efficacy of Chloral Hydrate in Outpatient Pediatrics for Sedation. Science Journal of Clinical Medicine. Vol. 9, No. 3, 2020, pp. 51-53. doi: 10.11648/j.sjcm.20200903.12

Received: June 30, 2020; Accepted: July 23, 2020; Published: August 5, 2020

\begin{abstract}
Objective: Sedation is frequently applied in children who need to undergo a diagnosis or treatment procedure. We aim to investigate the safety and efficacy of retention enema with chloral hydrate for the sedation of outpatient children. Methods: We retrospectively reviewed outpatient pediatric patients from March 1, 2020 to July 6, 2020, who were given an initial dose of chloral hydrate rectally for needed sedation. Patient demographics, sedation dose, onset action time, action duration time, adverse reactions were recorded. Results: In this study, totally fifty- four pediatric patients (36 males, 18 females; median [range] age, 3 [1-5] years) were sedated with chloral hydrate (median [range] dose, 70 [30-130] $\mathrm{mg} / \mathrm{kg}$ ). The median weight was 15 $(10-21) \mathrm{kg}$, and $48(88.9 \%)$ children was success to sedate and finished the designated examinations. Five cases (9.3\%) awoke quickly and one child (1.9\%) failure to fall asleep, which required rescheduling of sedation. The median action duration time of sedation was $3(1-8)$ hours, and all children recovered normally post-sedation. Only 2 cases $(3.7 \%)$ showed minor adverse reaction, presented as prolonged sleep. Conclusion: When strictly following the process, retention enema with chloral hydrate in pediatric patients for sedation in this study demonstrated a relatively high success rate and low risk of adverse reactions, and can be used safely and effectively for outpatient pediatrics.
\end{abstract}

Keywords: Chloral Hydrate, Sedation, Pediatrics

\section{Introduction}

Sedation is commonly used for painless diagnostic imaging and painful procedures of children, such as magnetic resonance imaging (MRI) examinations, electroencephalography tests, objective hearing tests, ophthalmic procedures, dental procedures, and so on. Qualified MRI images require fixed motion-free scan time to minimize artifacts. However, it is especially tough for children to keep motion-free for quite a long time. In order to obtain a high-quality imaging of pediatric patients, general anesthesia or sedation is routinely required [1]. General anesthesia can prevent patient movement and motion artifacts, and approximately $1.5 \%$ pediatric patients need to be underwent repeated examinations [2]. However, sedation is non-invasive compared to general anesthesia. The depth of sedation is divided into four levels (minimal sedation, moderate sedation, deep sedation and general anesthesia), according to American Society of Anesthesiologists [1]. It is noting that the sedation scale is a continuum. For the purpose of outpatient sedation, the goal is to achieve a certain level of sedation between moderate and deep sedation, in which the child requires repeated stimulation for response but spontaneous ventilations and cardiovascular function are maintained.

As one of the oldest sedative agents, chloral hydrate was discovered in 1832 and can be adequately absorbed orally and rectally [3]. After oral or rectal administration of chloral hydrate, the onset action time is about 30 to 60 minutes [4]. Adverse reactions of chloral hydrate are more frequently occurred in younger children which are younger than 6 months. Adverse reactions presented with hypotension, vomiting, desaturation, apnea, and prolonged sedation [5]. Chloral hydrate should be avoided in patients with severe hepatic insufficiency or moderate to severe renal dysfunction.

Most current studies about chloral hydrate oral solution for sedation were reported in recent years [6-9], but few about retention enema with chloral hydrate for sedation. In this study, we aim to investigate the efficacy and adverse reactions of 
retention enema with chloral hydrate for sedation of outpatient children.

\section{Methods}

The study was conducted in accordance with the principles of the Declaration of Helsinki. There is no link between the patients and the researchers, in addition, the de-identified data involve no potential risk to patients. We retrospectively reviewed outpatient pediatric patients from March 1, 2020 to July 6, 2020, who were given an initial dose of chloral hydrate rectally for needed sedation. All enrolled outpatient children underwent MRI scans or electroencephalograms by trained medical staff, following adequately sedation.

After sedation, the child's pulse, breathing, complexion and adverse reactions were noted. We contacted the parents by telephone on the following working day after sedation procedure with chloral hydrate. Patient' s current age, weight, sedation dose, onset action time, action duration time, adverse reactions (e.g. agitation, bradycardias, apnea, hypotension and prolonged sedation) were recorded. The length of time required for pediatrics to become fully awake was also recorded.

\section{Results}

Fifty-four pediatric patients (36 males, 18 females; median [range] age, 3 [1-5] years) were sedated with chloral hydrate (median [range] dose, 70 [30-130] $\mathrm{mg} / \mathrm{kg}$ ) in total. The median weight was $15(10-21) \mathrm{kg}$, and $48(88.9 \%)$ children was success to sedate and finished the designated examinations. Five cases $(9.3 \%)$ awoke quickly and one child (1.9\%) failure to fall asleep, which required rescheduling of sedation. The median action duration time of sedation was 3 (1-8) hours, and all children recovered normally post-sedation. Only 2 cases $(3.7 \%)$ showed minor adverse reaction, presented as prolonged sleep.

Table 1. Demographic distribution and sedation effects of pediatric patients $(n=54)$.

\begin{tabular}{ll}
\hline & Patients (n=54) \\
\hline Patient demographics & \\
Median age, years (range) & $3(1-5)$ \\
Male (n, \%) & $36(67)$ \\
Female (n, \%) & $18(33)$ \\
Median weight, kg (range) & $15(10-21)$ \\
Sedation with chloral hydrate & \\
Median dose, mg/Kg (range) & $70(30-130)$ \\
Median action duration time, h (range) & $3(1-8)$ \\
Success to sedate (n, \%) & $48(88.9)$ \\
Awoke quickly (n, \%) & $5(9.3)$ \\
Failure to fall asleep (n, \%) & $1(1.9)$ \\
Adverse reactions & $0(0)$ \\
Agitation (n, \%) & $0(0)$ \\
Bradycardias (n, \%) & $0(0)$ \\
Apnea (n, \%) & $0(0)$ \\
Hypotension (n, \%) & $2(3.7)$ \\
Prolonged sedation (n, \%) & \\
\hline
\end{tabular}

\section{Discussion}

Qualified MRI and electroencephalography examinations in young children play important roles in establishing an early diagnosis, which require a quiet, preferably motion-free time to avoid artifacts. It is still challenging to obtain painless diagnostic procedures for sedated outpatients to decrease patients' anxiety, movement, which ultimately promote the quality of procedural outcomes. The investigation can be conducted under natural sleep or drug-induced sleep or under general anesthesia. Chloral hydrate, midazolam, dexmedetomidine are recommended for sedation in children in the guideline $[10,11]$.

According to the children's anatomical characteristics of the rectum and anus, the average of rectal depth of infants and young children is $10 \mathrm{~cm}$. The mucosa above the dentate line and the rectal mucosa are full of venous plexus, therefore, when 5 to $10 \mathrm{~cm}$ depth of chloral hydrate retention enema is inserted, a rapid, stable and lasting sedative effect can be achieved. In this study, five cases $(9.3 \%)$ awoke quickly and one child $(1.9 \%)$ failure to fall asleep, which required rescheduling of sedation. The main causes of failure to fall asleep were failing to wake up early on the morning or keep awake on the way to the hospital.

When chloral hydrate retention enema is applied for the sedation of outpatient children, clinical nursing staff should strictly abide by the aseptic procedure. And the chloral hydrate should be given slowly, and kept for 3 to 5 minutes. The most frequent incident was prolonged sedation in this study. Prolonged sedation was occurred in two children $(3.7 \%)$, which lasted more than 6 hours, and West et al. also reported $1.33 \%$ of cases with prolonged sedation [12]. All cases only need to be monitored without any other intervention.

In general, there were no obvious clinical adverse effects presented in this study, and were similar to those currently reported [13-15]. However, several studies fund that the adverse effects of chloral hydrate can be bradycardia, apnea and decreased oxygen saturation [16]. These adverse effects tend to be more frequent in infants with lower birth weight [17]. In our study, the median age of the children was 3 years (range 1-5), and median weight was $15 \mathrm{~kg}$ (range 10-21), and few of them presented minor adverse reactions. Therefore, we speculate that the degree of adverse effects is related to age and weight, which requires the attention of clinical staff.

Chloral hydrate is safely and effectively by rectal administration, and it is well absorbed through gastrointestinal tract [18]. It can obtain a good sedative effect in a short time. The sedative effect of chloral hydrate is similar to physiological sleep, which has no obvious side effects. All children recovered normally post-sedation. As a clinical nursing operation technique and administration route, chloral hydrate retention enema is worthy of clinical promotion.

There are some limitations in our study. First of all, due to the retrospective nature of this study, part of the information must be obtained from the parents of the child, so the sample size of this study is relatively small. Therefore, we need to expand the sample size in future studies to verify the 
conclusion. In addition, as the sedation method of pediatric patient in our central injection room is mostly chloral hydrate retention enema, it is lack of comparison with other sedation methods and further research is needed.

\section{Conclusion}

In conclusion, for children who cannot cooperate, the use of chlorine hydrate is recommended for painless examinations, clinical diagnosis and treatment. From our perspective, the advantage of chloral hydrate sedation is that we can benefit from a relatively free time. The results of the study indicate that chloral hydrate is a sedative for children in MRI and electroencephalography services when strict patient selection criteria are met. Retention enema with chloral hydrate in pediatric patients for sedation in this study demonstrated a relatively high success rate and low risk of adverse reactions, and can be used safely and effectively for outpatient pediatrics but under specialized administration.

\section{Conflicts of Interest}

The authors declare that they have no conflicts of interest.

\section{References}

[1] Delgado J, Toro R, Rascovsky S, et al. Chloral hydrate in pediatric magnetic resonance imaging: evaluation of a 10-year sedation experience administered by radiologists. Pediatr Radiol. 2015; 45 (1): 108-114.

[2] Malviya S, Voepel-Lewis T, Eldevik OP, et al. Sedation and general anaesthesia in children undergoing MRI and CT: adverse events and outcomes. Br J Anaesth. 2000; 84 (6): 743-748.

[3] Ratnapalan S. Chloral hydrate sedation in children. Clin Pediatr (Phila). 2014; 53 (10): 933-936.

[4] Treluyer JM, Andre C, Carp PF, et al. Sedation in children undergoing CT scan or MRI: effect of time-course and tolerance of rectal chloral hydrate. Fundam Clin Pharmacol. 2004; 18 (3): 347-350.

[5] Heistein LC, Ramaciotti C, Scott WA, Coursey M, Sheeran PW, Lemler MS. Chloral hydrate sedation for pediatric echocardiography: physiologic responses, adverse events, and risk factors. Pediatrics. 2006; 117 (3): e434-e441.

[6] Chen Z, Lin M, Huang Z, et al. Efficacy of chloral hydrate oral solution for sedation in pediatrics: a systematic review and meta-analysis. Drug Des Devel Ther. 2019; 13: 2643-2653.
[7] Joo EY, Kim YJ, Park YS, et al. Intramuscular dexmedetomidine and oral chloral hydrate for pediatric sedation for electroencephalography: A propensity score-matched analysis. Paediatr Anaesth. 2020; 30 (5): 584-591.

[8] Cheng X, Chen Z, Zhang L, et al. Efficacy and Safety of Midazolam Oral Solution for Sedative Hypnosis and Anti-anxiety in Children: A Systematic Review and Meta-Analysis. Front Pharmacol. 2020; 11: 225.

[9] Yuen VMY, Cheuk DKL, Hui TWC, Wong ICK, Lam WWM, Irwin MG. Oral chloral hydrate versus intranasal dexmedetomidine for sedation of children undergoing computed tomography: a multicentre study. Hong Kong Med J. 2019; 25 Suppl 3 (1): 27-29.

[10] 2018 surveillance of sedation in under 19s: using sedation for diagnostic and therapeutic procedures (NICE guideline CG112). London: National Institute for Health and Care Excellence (UK); 2018.

[11] Mace SE, Brown LA, Francis L, et al. Clinical policy: critical issues in the sedation of pediatric patients in the emergency department. J Emerg Nurs. 2008; 34 (3): e33-e107.

[12] S. K. West, B. Griffiths, Y. Shariff, et al. Utilisation of an outpatient sedation unit in paediatric ophthalmology: safety and effectiveness of chloral hydrate in 1509 sedation episodes. Br. J. Ophthalmol. 2013; 97 (11) 1437-1442.

[13] Necula V, Stamate MC, Blebea C, Cozma S. Safety and effectiveness of chloral hydrate in outpatient paediatric sedation for objective hearing tests. Int $\mathrm{J}$ Pediatr Otorhinolaryngol. 2019; 126: 109605.

[14] Karaoui M, Varadaraj V, Munoz B, et al. Chloral Hydrate Administered by a Dedicated Sedation Service Can Be Used Safely and Effectively for Pediatric Ophthalmic Examination. Am J Ophthalmol. 2018; 192: 39-46.

[15] Finnemore A, Toulmin H, Merchant N, et al. Chloral hydrate sedation for magnetic resonance imaging in newborn infants. Paediatr Anaesth. 2014; 24 (2): 190-195.

[16] Allegaert K, Daniels H, Naulaers G, et al. Pharmacodynamics of chloral hydrate in former preterm infants. Eur J Pediatr. 2005; 164 (7): 403-407.

[17] Litman RS, Soin K, Salam A. Chloral hydrate sedation in term and preterm infants: an analysis of efficacy and complications. Anesth Analg. 2010; 110 (3): 739-746.

[18] Cozzi G, Norbedo S, Barbi E. Intranasal Dexmedetomidine for Procedural Sedation in Children, a Suitable Alternative to Chloral Hydrate. Paediatr Drugs. 2017; 19 (2): 107-111. 